\title{
The method of decomposition domain for the digital modelling of a jet
}

\author{
Khalid Adnaoui ${ }^{1, *}$, Nourdine Tounsi ${ }^{1}$, Mohamed Chagdali ${ }^{2}$, Soumia Mordane ${ }^{2}$ \\ ${ }^{1}$ Département de Mathématique \& informatique Faculté des Sciences Ben M'Sik, Casablanca, Morrocco \\ ${ }^{2}$ Département de Physique, Faculté des Sciences Ben M'Sik, Casablanca, Morrocco
}

\section{Email address:}

Khalid.adnaoui@gmail.com (K. Adnaoui)

\section{To cite this article:}

Khalid Adnaoui, Nourdine Tounsi, Mohamed Chagdali, Soumia Mordane. The Method of Decomposition Domain for the Digital Modelling of a Jet. American Journal of Applied Mathematics. Vol. 2, No. 2, 2014, pp. 64-68. doi: 10.11648/j.ajam.20140202.14

\begin{abstract}
This work concerns to the digital treatment of the problems in strong nonlinearity during the resolution of the equations of Navier Stokes, in particular those due to the strong recirculation in turbulent regime. The developed idea is to use the method of subdomains. The domain of the flow is decomposed into several sub domains separated by fictitious borders. In each of these sub domains, we use the best digital adapted method. The passage in the entire domain is made by digital connecting. This connecting is made by covering of domain. The results are presented in the case of a jet of rejection emitted by the bottom in a rectangular canal. In this application, we divided the domain of study into two parts: Meadows of the limit coat, we use the method of the finished differences and in the outside zone the resolution is made by the particular method.The fictitious interface between these two subdomains is processed by the method particles - meshing. A validation of this approach is made by comparison with a direct calculation in entire domain.
\end{abstract}

Keywords: Equations of Navier Stocks, Digital Methods, Particular Methods, Method of Finished Differences, Decomposition of the Domain, Schema T.S.C of Projection Interpolation

\section{Introduction}

In this work, we present a complete numerical solution of the flow map generated by a jet emitted from the bottom in an open channel. The fluid is considered to be an incompressible viscous. The direct solution of such a problem by finite difference methods poses a number of challenges for large Reynolds numbers. Indeed, digital instabilities due to the treatment of the nonlinear terms in zones with strong variations of speed, appear what requires to refine the meshing. But this type of methods allows to take into account the conditions in the limits on the solid walls. For large numbers of Reynolds, other methods are better adapted to handle the flows of viscous fluid in the case of the recirculation strong vectors speeds, we quote the particular methods. But they present other types of inconveniences on the treatment of the conditions to the limits in the wall.

We present a numerical approach that combines the advantages of both methods mentioned above. For this, the domain of the flow is divided into two sub-domains in each of which is used the most appropriate method: In the neighbourhood of walls, we use the method of the finished differences and somewhere else the particular method. But it raises the problem of connecting both sub-domains, that is of the transmission of the information enter a method which uses a meshing (the method of the finished differences) and another one without meshing (the particular method). To solve this problem, we used the following technique:

We proceeded to the emission of particles after a test on the sign of the speed normal for the interface separating both under domains. Particles that enter by recirculation effect in the first subdomain close to the solid wall are distributed on the meshing of differences finished by a technique particle - meshing and new particles are created in exchange [1],[2].

\section{Presentation of Method}

The application of the decomposition method of the domain for a numerical solution of the Navier-Stokes equations is done in several steps: 


\section{Step 1: geometric decomposition of the domain:}

The domain resolution is decomposed into several subdomains. This decomposition is based on physical and numerical considerations: Nature of the flow regime, ease of writing the conditions in the limits and treatment of nonlinearities in the equations of movement.

For connexion domain, the decomposition is based on the partitioning of the domain $\Omega$ into $\mathrm{n}(\mathrm{n} \geq 2)$ subdomain $\Omega i$ :

$$
\Omega=\bigcup_{\mathbf{1} \mathbf{I}_{\mathbf{s}} \mathbf{n}} \mathbf{\Omega}_{\mathbf{1}}
$$

The intersection between the subdomain is limited to the interfaces (Figure 1):

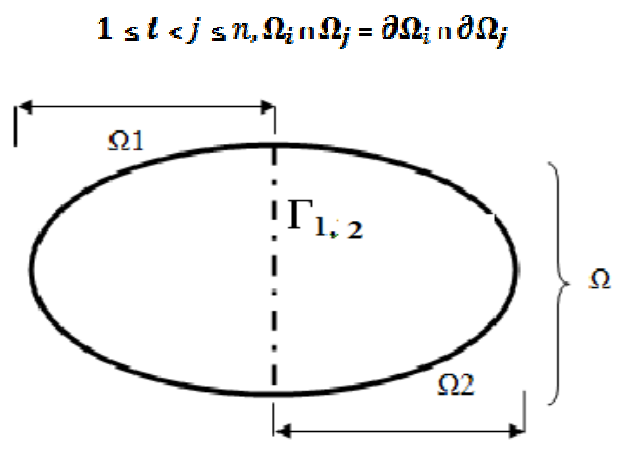

Figure 1. Partition into two subdomains $\Omega 1$ et $\Omega 2$.

\section{Step 2: Choice of method for solving in the subdomain:}

The choice of the method of resolution in every subdomain is made on the basis of the capacity of the digital method in treated the equations of the movement. In particular, we consider the calculation time, the adaptation to the treatments of non-linearity and to the geometrical configuration.

\section{Step 3: Writing conditions interfaces:}

It is a question of writing conditions of continuity of variables between both under geometrical domains. In particular it is necessary to assure the implicit character of the resolution of the equations of movements.

\section{Application}

We tried to solve the equations of Navier-Stokes and the equation of transport of mass of a jet at the bottom of a canal. In terms of vortex function $\omega$, function of current $\psi$, and concentration $C$, these equations are [1]:

$$
\begin{gathered}
\frac{\partial w}{\partial t}+(\vec{U} \cdot \vec{\nabla}) w=\frac{1}{\mathcal{R} e} \Delta w+\frac{1}{\mathcal{F} \mathrm{r}^{2}}\left[\vec{\nabla} C \wedge \frac{\vec{g}}{\|\vec{g}\|}\right] \cdot \vec{k} \\
\Delta \psi=-w
\end{gathered}
$$

$$
\begin{aligned}
& \vec{U}=\vec{\nabla} \wedge(-\psi \overrightarrow{\mathrm{k}}) \\
& \frac{\partial C}{\partial t}+(\vec{U} \cdot \vec{\nabla}) C=\frac{1}{\mathcal{R e} \mathcal{P} r} \Delta C
\end{aligned}
$$

With the following notations:

- $\vec{\nabla}$ et $\Delta$ are respectively the gradient operator and the Laplacian operator.

- $\quad \mathrm{t}$ is the time variable.

- $\vec{k}$ is c the directly perpendicular vector in the plan of the flow.

- $\vec{U}$ Is the velocity Victor.

- $\psi$ is the function of current

- $\quad \omega$ is the vortex function, such a $\vec{\omega}=\omega \vec{k}=\vec{\nabla} \wedge \vec{U}$

- $\mathrm{C}$ is the concentration of the pollutant.

- $\quad \vec{g}$ acceleration of gravity.

- $\mathfrak{R} e, \operatorname{Pr}, F r$ Represent respectively the numbers of: Reynolds, Prandtl and Froude.

The connected rectangular domain $\Omega$ is decomposed intotwo sub-domains $\Omega 1$ and $\Omega 2$ separated by a border $\Gamma$ (figure 2):

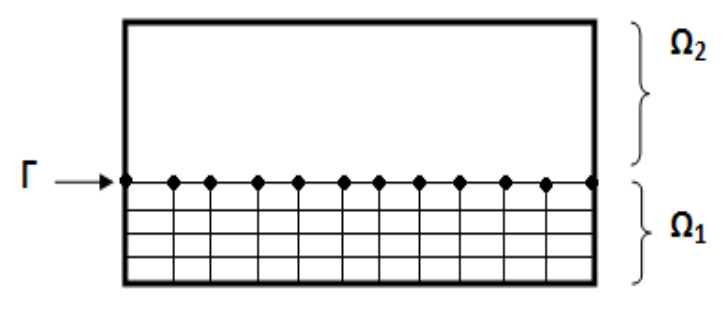

Figure 2. Subdivision of the domain $\Omega$.

The jet is the bottom (Domain $\Omega 1$ ) and the flow is dominated by the effect of the walls. The resolution method selected in this subdomain is the method of finite differences [3]. It is the method Eulerienne which possesses the advantage to facilitate the writing of the conditions in the limits on the solid walls. Far from walls (Domain $\Omega 2$ ), the flow is external in large number of Reynolds and in big recirculation of speed. In this domain, we use the particular method [4] , It is the lagrangienne method which consists to discretize the transportable variables by means of a number of particles which will be followed in their movement..

Between both sub-domains the interface is the place of transmission the results of resolution by a method which uses a meshing (the method differences finis on $\Omega 1$ ) and a method without meshing (the particular method on $\Omega 2$ ), requires special treatment.

A first algorithm is to transmit information according to the sign of the velocity normal to the boundary between the two sub domains $\Omega 1, \Omega 2$ without recovery domain. This is an explicit approach but is used here for the implementation of the method presented in this work.made for all the domain of Study by the finite difference method. 


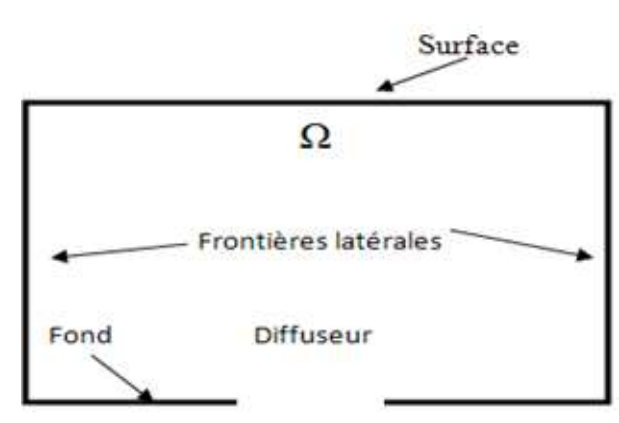

Figure 3. Domain of study $\Omega$.

\section{Results}

Geometric data: Length $\mathrm{L}=6 \mathrm{~m}$ and the height $\mathrm{l}=4 \mathrm{~m}$.

The fictitious interface separating both under domains $\Omega$ 1 and $\Omega 2$ is fixed in $\mathrm{y}=1.4$

It is from this interface that are emitted particles representing the jet flow in the subdomain $\Omega 2$ betweeny $=$ 1.4 and $\mathrm{y}=5$.

The results will be presented in terms of ISO- values of the vortex function $\omega$, function of Current $\psi$, and concentration $\mathrm{C}$.

Physical data $F_{r}=18, P_{r}=1.5$.

In the figure 4-a, 4-b, 5-a, 5-b, 6-a, 6-b, we present the results of calculate direct who was

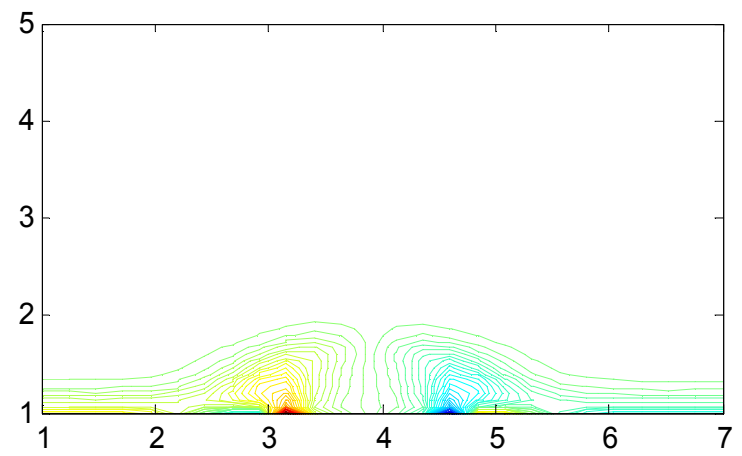

Figure 4-a. ISO - values of the vortex function by direct calculation with, $t=1, \Re_{e}=100$.

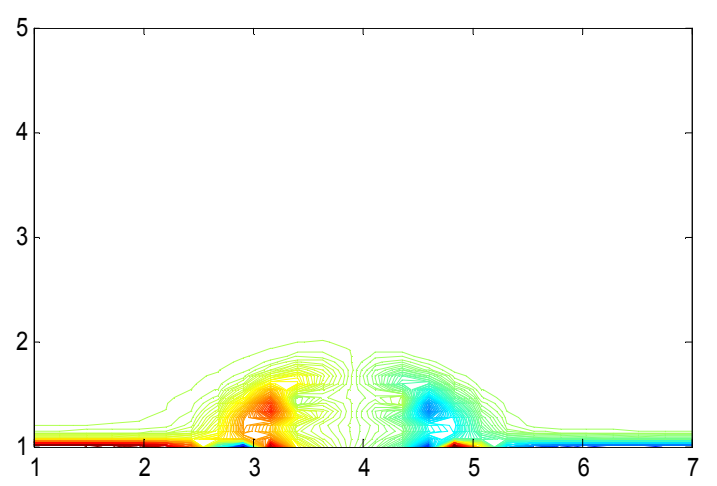

Figure 4-b. ISO - values of the vortexfunction by direct calculation with, $t=1, \Re_{e}=1000$.

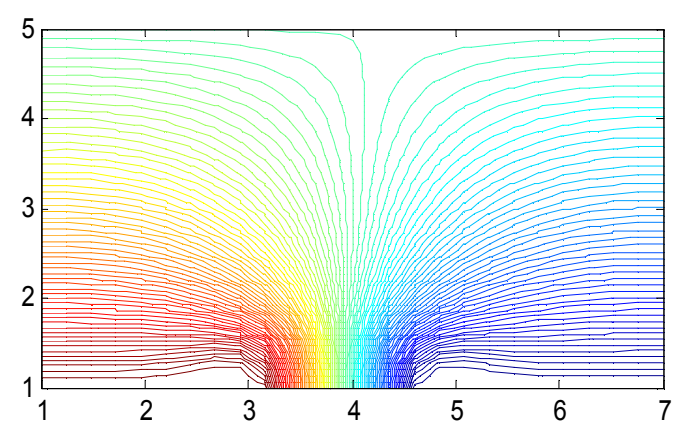

Figure 5-a.ISO - values of the current function by direct calculation with, $t=1, \mathfrak{R}_{e}=100$.

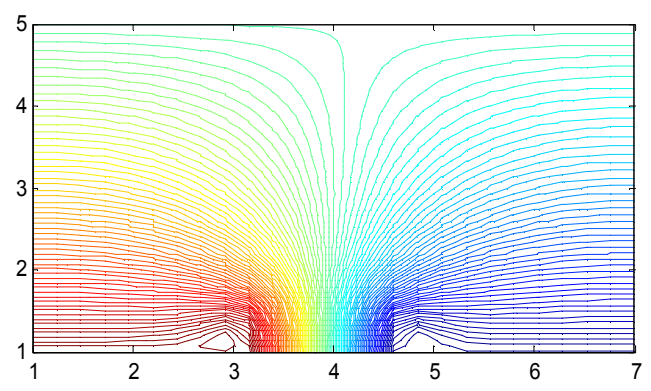

Figure 5-b. ISO - values of the current function by direct calculation with, $t=1, \Re_{e}=1000$.

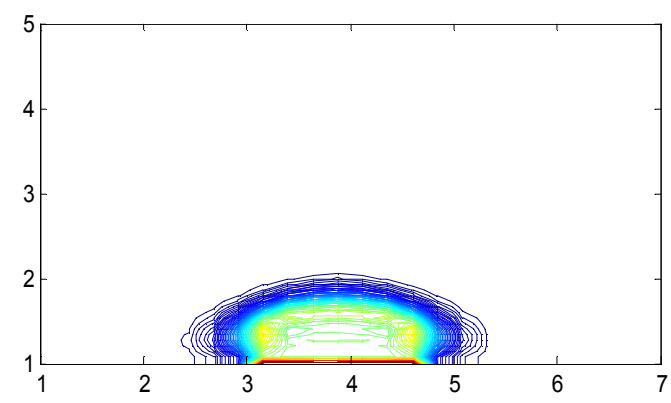

Figure 6-a. ISO-Concentration by direct calculationwith, $t=1, \Re_{e}=100$.

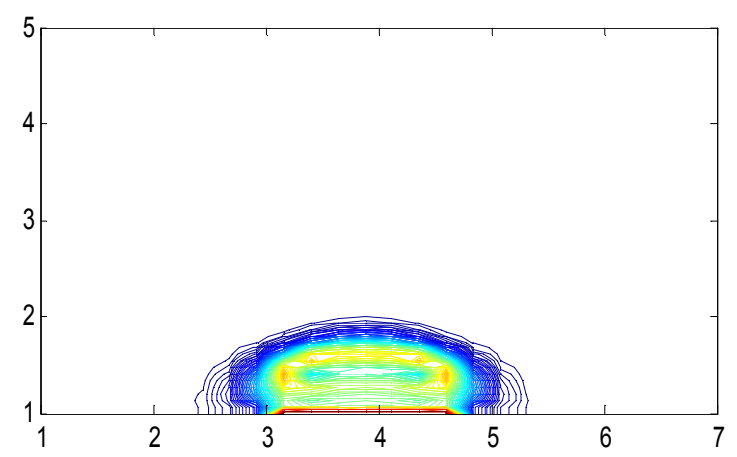

Figure 6-b. ISO-Concentration by direct calculation with, $t=1, \Re_{e}$ $=1000$.

In the same geometrical configuration, we solved the complete equations of Navier-Stokes by the method of subdomains. 


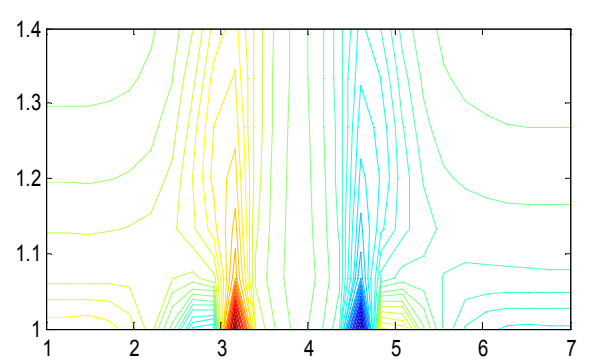

Figure 7-a. ISO - values of the vortex function In subdomain $\Omega 1$ with $\Re_{e}$ $=100$ et $t=1$.

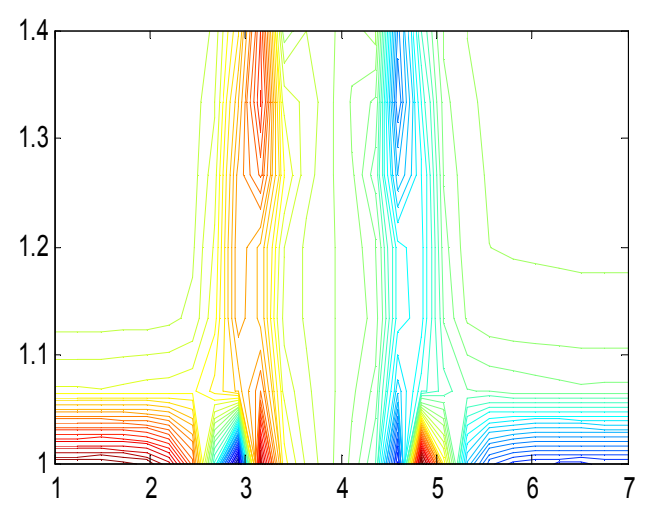

Figure 7-b. ISO - values of the vortex function In subdomain $\Omega 1$ with $\mathfrak{R}_{e}$ $=1000$ et $t=1$.

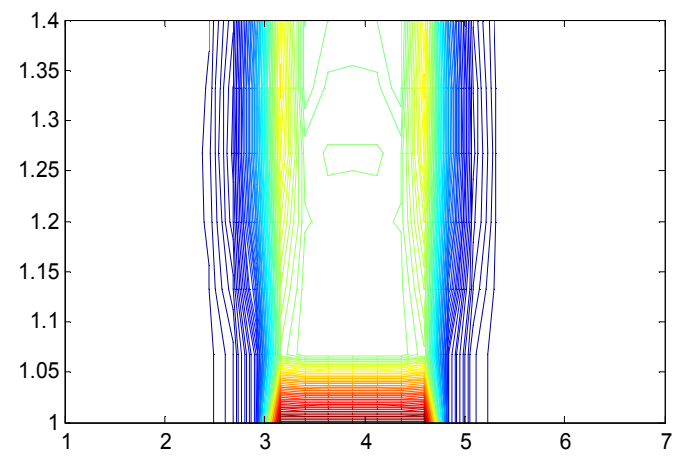

Figure 8-a. ISO-ConcentrationIn subdomain $\Omega 1$ with $\Re_{e} 100$ et $t=1$.

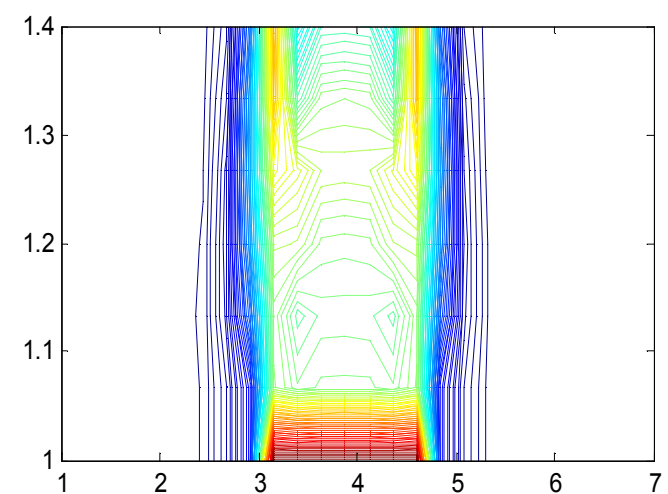

Figure 8-b. ISO-ConcentrationIn subdomain $\Omega 1$ with $\Re_{e} 1000$ et $t=1$.

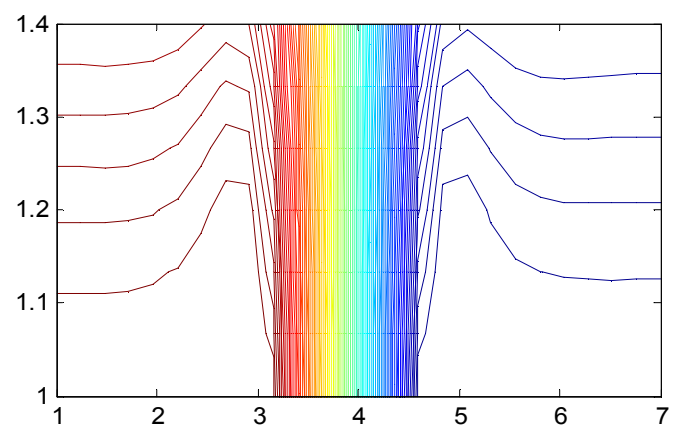

Figure 9-a. ISO - values of the current functionIn subdomain $\Omega 1$ with $\Re_{e}$ $=100$ et $t=1$.

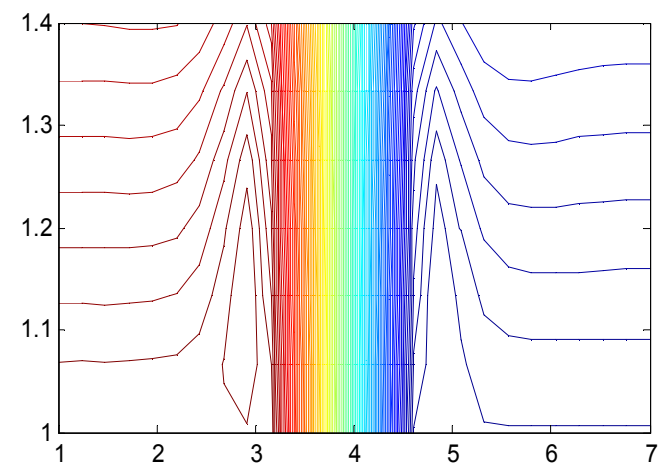

Figure 9-b. ISO - values of the current functionIn subdomain $\Omega 1$ Avec $\mathfrak{R}_{e}$ $=1000$ et $t=1$.

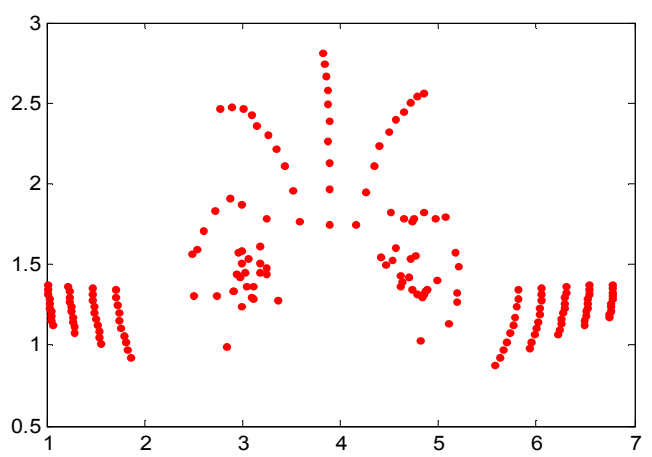

Figure 10-a. Movement of fluid particlesin the subdomain $\Omega 2$, avec $\mathfrak{R}_{e}$ $=100, t=1$.

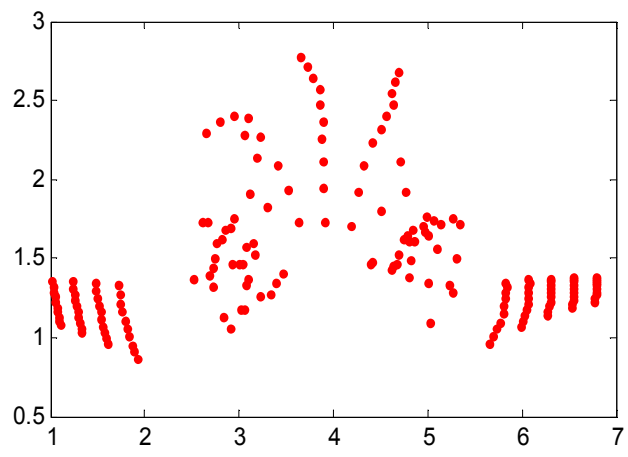

Figure 10-b. Movement of fluid particlesin the subdomain $\Omega 2$, avec $\mathfrak{R}_{e}$ $=1000, t=1$. 
The Figures (8-a, 9-a, 10-a) show a good continuity of the phenomenon on both sides of the interface.

For high Reynolds number the method of decomposition domain reproduces the jet flow, with fewer difficulties than those encountered with the direct method (Figure 7-b, 8-b, 9-b, 10-b).

\section{Conclusions}

From the results presented, we can conclude that the method of multi-domain has removed the difficulties posed by the finite difference method, and which are related to numerical instabilities related high Reynolds numbers. Based on the combination of several methods.

The multi-method domain allows to use the advantages of a digital method given in the region where it is the most effective.

\section{References}

[1] A. Jolles, Résolution des équations de Navier Stokes par une méthode particules-maillage, Thèse de Doctorat Université Paris-VI, Décembre 1989.

[2] G.Mangoub, Etude numérique de l'interaction tourbillon paroi par une méthode particule-maillage, thèse de Doctorat Université paris -VI, Décembre 1992.

[3] O. DAUBE - T. P. LOC," Etude numérique d'écoulements instationnaires de fluides visqueux incompressibles autour de corps profilés, par une méthode combinée d'ordre $\mathrm{O}(\mathrm{h} 2)$ et $\mathrm{O}(\mathrm{h} 4)$ Journal de mécanique Vol 17 n6 1978.

[4] S. Huberson, Modélisation asymptotique et simulation numérique d'écoulements tourbillonnaires, Thèse de Doctorat d'Etat, Décembre 1986 